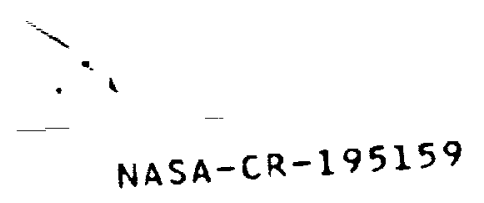

$$
N 15
$$

UPTICAL MATERLALS

$$
\text { NAG } 955
$$

$$
1 N-76-C R
$$

\title{
Excited state dynamics of thulium ions in Yttrium Aluminum Garnets
}

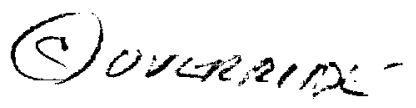

\author{
G. Armagan. A.M. Buoncristiani

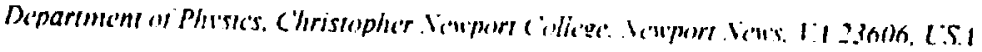

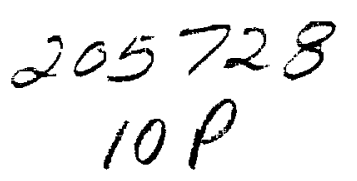

and

\author{
B. Di Bartolo \\ Department of Phisics. Boston College. Chesinut Hii. Il.t u2loi. LS.t
}

Received is June 1991: revised manuscript recesved + Siptember 1991

\begin{abstract}
The processes that take place in the excited states of a trivalent Thulium (Tm) ion in an Yurium Aluminum Gamet (YAG) crystal. being relevant to the use of this system for laser applications. have been the object of several studies. We have reexamined this system. locusing our attention on the dynamics of $\mathrm{Tm}$ iollowing its excitation in the ${ }^{3} \mathrm{H}_{4}$ level. Under these conditions the system relaxes through a cross-relaxation process. ${ }^{3} \mathrm{H}_{4} \rightarrow{ }^{\prime} \mathrm{F}_{4},{ }^{3} \mathrm{H}_{6} \rightarrow{ }^{3} \mathrm{~F}_{4}$. whose rate depends upon both the concentration of the $\mathrm{Tm}$ ion and the temperature of the crystal. The excitation spectrum obtained by monitoring the $1.8 \mu \mathrm{m}$ emission of $\mathrm{Tm}$ (due to the ${ }^{3} \mathrm{~F}_{4} \rightarrow{ }^{3} \mathrm{H}_{6}$ (ransition ) indicates an increase in the contribution to this emission from the ${ }^{3} \mathrm{H}_{4}$ level relative to the ${ }^{3} \mathrm{H}_{3}$ level as the ingly the duration of the luminescence the increased role played by the ${ }^{3} \mathrm{H}_{4}$ level in pumping the infrared emission. Correspondtration quenching of this lifetime can be fil ating in the ${ }^{\mathrm{J}} \mathrm{H}_{4}$ level is shortened as the concentration of $\mathrm{Tm}$ increases. The concention: from this fit the intrinsic Tm lifetime in absenel which assumes that the cross-relaxation is due to a dipole-dipole interacthe rate of the cross-relaxation process. We have evaluaed cross relaxation can be derived. We have used this lifetime to calculate We have also calculated the microscopic have evaluated this rate as function of temperature and found it to be fastest at $77 \mathrm{~K}$. imental features: (i) the time evolution interaction parameters for the cross-relaxation process by using two independent experemission and the ${ }^{3} \mathrm{H}_{\mathrm{b}} \rightarrow{ }^{\prime} \mathrm{F}_{4}$ absorption. We have a and calculated the relevant asorption. We have also considered the migration of excitation among the $\mathrm{Tm}$ ions in the ${ }^{3} F_{4}$ level

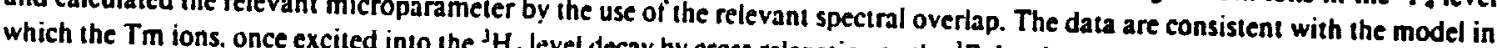
to other Tm ions.
\end{abstract}

\section{Introduction}

The first detailed studies of the optical properties of solids doped with rare earth ions confirmed that energy transter among such ions by various resonant inter-ionic interactions was possible: subsequent investigation showed that these processes could even produce luminescence with quantum yields greater than unity [1]. The possibility of using these energy Iransier processes to enhance the characteristics of solid state lasers pumped by flash lamps was also recognized early [2]: the sensitization of a laser medium by optically active ions was used to increase the pump etficiency and lower the threshold [3]. Later. with the emergence of laser-pumped lasers, where the pump radiation populates only one manifold and this excitation finds its way efficiently to lasing levels by one or more transter process, it became important to understand these energy transfer mechanisms in more detail.

$\mathrm{Tm}^{\mathrm{j}+}$ in crystalline solids has been used in several important laser applications. The interest in this ion arises mainly from two facts: (i) it has a strong absorption $\left({ }^{3} \mathrm{H}_{n} \rightarrow{ }^{\prime} \mathrm{H}_{4}\right)$ in the region where pumping with semiconductor diode lasers can be used [4], and (ii) it efficiently accepts energy transierred from $\mathrm{Cr}$, thus tacilitating broadband pumping $[5,6] . \mathrm{Tm}^{3+}$ in $Y$ IG has been shown to lase [7]: in addition this ion 
has been used as a sensitizer for Ho [8.9]. I diodepumped Tm.Ho: YAG laser has been demonsirated by Kintz et al. [10]. Much of this work has indicated the presence of a complex sequence of energy iransier and migration events: these phenomena. being critical to the lasing process either by providing transter of excitation energy to lasing ions or by producing loss mechanisms. continue to call the attention of workers in the field. A group of researchers at the University of Lyon has conducted a study of such processes involving $\mathrm{Tm}$ and $\mathrm{Ho}$ in $\mathrm{LiYF}_{\downarrow}$ (YLF) [11]. We are presently conducting a thorough invesligation of $\mathrm{Cr}$. Tm and $\mathrm{Ho}$ in Y.AG.

As part of this effort we have investigated the dynamical processes that take place when $\mathrm{Cr}$ and $\mathrm{Tm}$ are present in a $Y A G$ crystal $[5,6]$. The present work is a logical extension of this previous investigation in that it discusses the processes that take place within the $\mathrm{Tm}$ system in YAG, once it has been provided with some excitation energy. In particular. we shall treat the phenomenon of cross relaxation that takes place when the $\mathrm{Tm}$ ion is excited to its ${ }^{3} \mathrm{H}_{4}$ level. This phenomenon has been studied by other workers. some of them using spectroscopic measurements [12], and others laser measurements [13]. We ourselves have dealt with it in the past at various times [14.15]. We shall also address our attention to the energy transfer processes that may take place among Tm ions in their lowest excited ${ }^{3} F_{+}$level. In the present article we would like to present some additional data and. in the process of doing so. treat in a comprehensive way the whole problem of energy transfer in this system.

The most informative work on the specira and energy levels of $\mathrm{Tm}^{3+}$ in YAG is due to Gruber et al. [16], who studied the absorption and emission spectra of this system. The energy level diagram of lig. I is merely indicative. in the sense that it reports only the approximate positions of the centroid for each $2 s+1 L$ manifold. For greater detail one should look at the work just mentioned.

\section{Samples and experimental}

Samples of YAG:Tm. grown by the Union Carbide Corporation with various $T m$ concentrations ranging from 0.75 to $6 \%$. were examined. A typical sample had the dimensions $1.0 \times 1.0 \times 0.5 \mathrm{~cm}$. Some of the

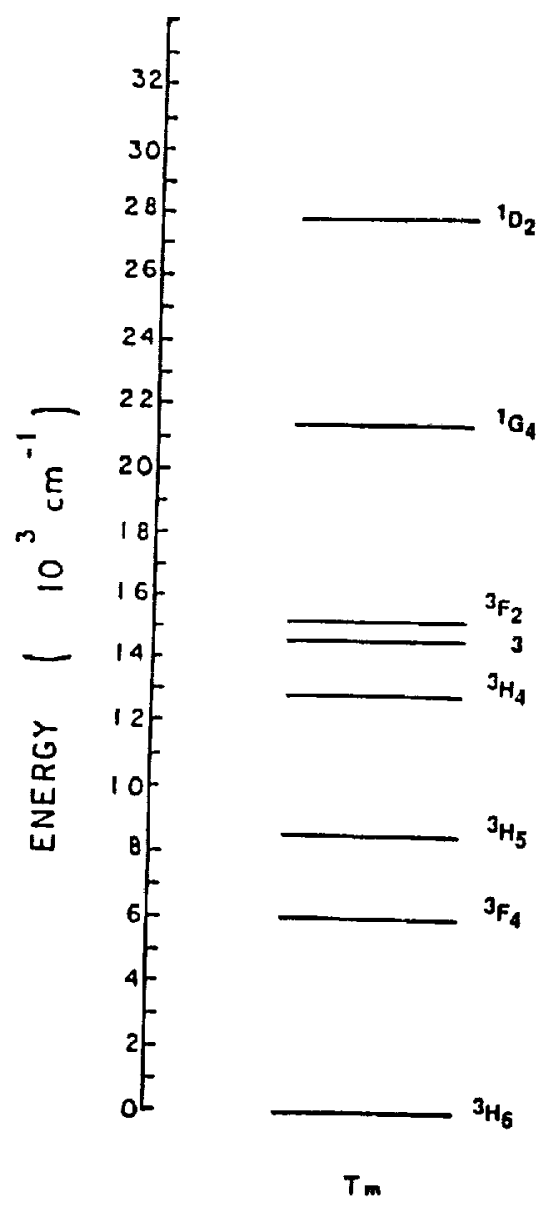

Fig. 1. Energy levels of $\mathrm{Tm}^{\mathrm{d}+}$ in YAG.

samples were co-doped with $\mathrm{Cr}$, but the presence of the $\mathrm{Cr}_{r}$ ions was not expected to influence the study of the processes within the Tm system.

The room temperature absorption spectra of the samples were obtained by using a Perkin Elmer Corp. Model Lambda 9 spectrophotometer. The low-temperature absorption spectra were measured in transmission with the same equipment used for the luminescence measurements and described below.

The following sources were available to excite the samples in order to obtain the luminescence spectra: a Jarrell Ash $30 \mathrm{~W}$ tungsten halogen lamp, a Sharp Corp. Model LTO24MFO diode laser, and a Coherint Inc. Model 526 Ar ion laser. The luminescence was observed at 90 degrees to the direction of excitation. focused onto the entrance slit of a Spex Industries Model 1269. 126 meter scanning monochroma- 
tor. The signal in the optical region was detected by a RC. A Model C31034 photomultiplier ( having a Gats photocathode. and cooled by a Haake Buchler Instruments. Inc. Model KI refrigerator). amplified and discriminated by a Spex Industries Model DM 102 Photon Counting Input Module. The signal in the infrared region was detected by a Spex Industries Model 1428 lead sulfide detector and amplified by a Stanford Research Systems Model SR510 lock-in amplifier. Both signals were processed and stored by a Spex Industries Model DM I B spectrometer controller and data processor. Radiation trapping effects were minimized by pumping the samples close to the suriace from which luminescence was observed so that the emitted light traveled only a short distance in the sample.

The excitation spectra were obtained by selecting and varying the exciting wavelength by means of a Spex Industries Model 1681. $0.22 \mathrm{~m}$ monochromator. The pulsed luminescence measurements were made by using a Quantel International Model TDL51 tunable dye laser pumped by Quantel International Model $660 \mathrm{~A}-10 \mathrm{Nd}$ :YAG Laser. The length of the pulses produced by these lasers $(\sim 10 \mathrm{~ns})$ was much shorter than the decay times measured. The signal detected by either the photomultiplier or a Judson Infrared Inc. Model JI2TE2 In.As detector was amplifited by an Analogic Data Precision D 1000 dual amplifier, processed and stored by an Analogic Data Precision Data 6000 waveform analyzer.

The samples were mounted in either a Janis $\mathrm{Re}$ search Co., Inc. Model 8DT cryostat or a CTI-Cryogenics Model 12 cold head connected to a Model SC-21 compressor. The sample temperature was varied from $810400 \mathrm{~K}$ by using either a Lake Shore Cryotronics Model DRC-80C or a Spectra Physics Model 5720 digital thermometer/controller.

\section{Experimental results}

\section{I. .Ibsorption. luminescence, and excitation spectra}

The absorption spectrum of YAG:Tm(1\%) obtained at $295 \mathrm{~K}$ from 300 to $2600 \mathrm{~nm}$ shows bands in correspondence to the transitions from the ${ }^{3} \mathrm{H}_{6}$ ground level to the ' $D_{2}$, ' $G_{4}$. ' $F_{2},{ }^{3} F_{3},{ }^{\prime} H_{4},{ }^{3} H_{5}$, and ' $F_{4}$ levels. Our data are not presented here. on account of the more complete measurements made by Gruber et al. and reported in ref. [16].

The luminescence spectra of Y.AG:Tm(1\%) in the infrared region at 550.300 and $78 \mathrm{~K}$ are shown in fig. 2: these measurements were made by exciting the sample with a tungsten lamp. The spectral output of this sample includes also a much weaker infrared emission covering the region 1300 to $1550 \mathrm{~nm}$ : this emission at room temperature is reported later in this article (in fig. 11).

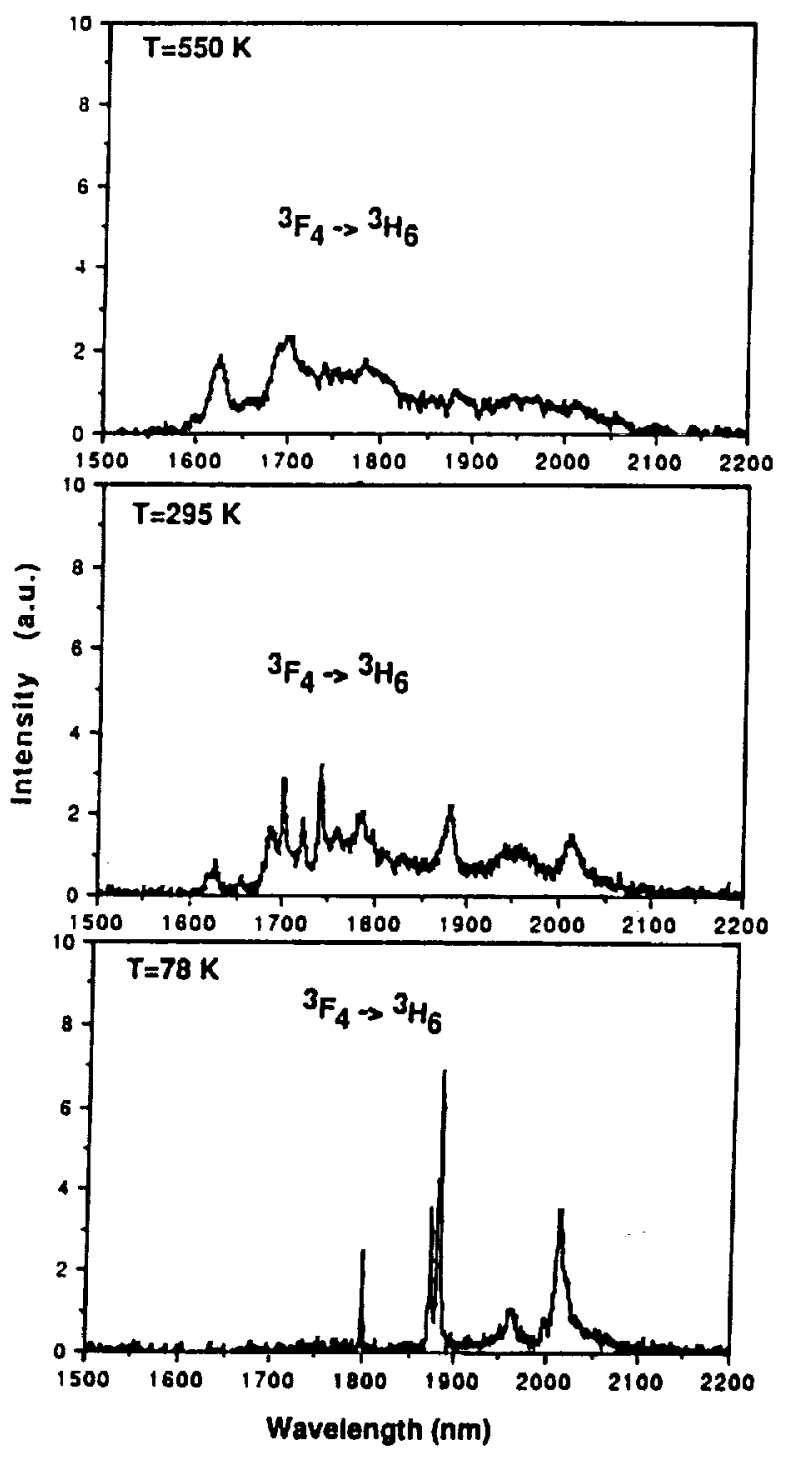

Fig. 2. Inirared luminescence of Y.AG:Tm (1\%) at 78. 295. and $550 \mathrm{~K}$. The region 1.5 to $2.1 \mu \mathrm{m}$ was covered and a $\mathrm{W}$ source was used. 
In addition. other measurements were run at room tomperature by exciting the sample with a tungsten lamp. or by means of an Ar laser. Excitation by the latter at $460 \mathrm{~nm}$ resulted in the pumping of level ' $G_{4}$ and produced evidence of ${ }^{\prime} \mathrm{G}_{4} \rightarrow{ }^{3} \mathrm{H}_{5}$ and ${ }^{1} \mathrm{H}_{4} \rightarrow{ }^{3} \mathrm{H}_{6}$ transitions. The study of these additional transitions. not reported in ref. [16]. is not relevant to the dynamics of $\mathrm{Tm}$ ions following their excitation to the ${ }^{\prime} H_{4}$ level and will be pursued and reported later.

The room temperature excitation spectra obtained by monitoring the emission from the ${ }^{3} \mathrm{~F}$, manifold for Iwo samples: YAG:Tm(0.75\%) and YAG:Tm(6\%) are shown in tig. 3 . We note the increase in intensity of the peaks corresponding to the ' $F_{:}$. ' $F_{1}$ and ' $H_{4}$ levels relative to the peak corresponding to the ${ }^{H}$; level. with increasing $\mathrm{Tm}$ concentration. This is a clear indication of the increased transter of excitation within the Tm system. from the ${ }^{:} \mathrm{H}_{4}$ to the : $F_{4}$ manifolds.

\subsection{Response to pulsed excitation}

The decay curves of the Tm emission from the ${ }^{3} \mathrm{H}_{4}$ level at room temperature and for several $\mathrm{Tm}$ concentrations are shown in fig. 4. For the lowest $\mathrm{Tm}$ concentration $(0.75 \%)$ the decay pattern is exponential, except at very early times $(t<50 \mu \mathrm{s})$. As the Tm concentration increases the curves assume the shapes generally associated with the decay of a sensitizer in the presence of an increasing number of activators: these curves are very similar to the ones obtained by Becker et al. [17].

If we scale all of the curves so that they have an amplitude $l$ at $t=0$. then the area under an individual curve gives a measure of the duration of that particular decay. We call the value of this area the "lifetime" of the ${ }^{3} \mathrm{H}_{4}$ level; the variation of this lifetime with $\mathrm{Tm}$ concentration is reported in lig. 5 .

We have also measured the temperature dependence of the lifetimes as detined above. Fig. 6 shows typical decay curves at 300 and $77 \mathrm{~K}$. for the samples with $2 \%$ and $6 \% \mathrm{Tm}$ concentrations. The results for all the samples examined in the 8 to $350 \mathrm{~K}$ range are shown in fig. 7 . For the $0.75 \% \mathrm{Tm}$ concentration the lifetime is almost constant with temperature. For higher Tm concentrations the lifetimes decreases from 8 to $77 \mathrm{~K}$. then starts to increase, and above $200 \mathrm{~K}$ remains practically constant. This behavior results in

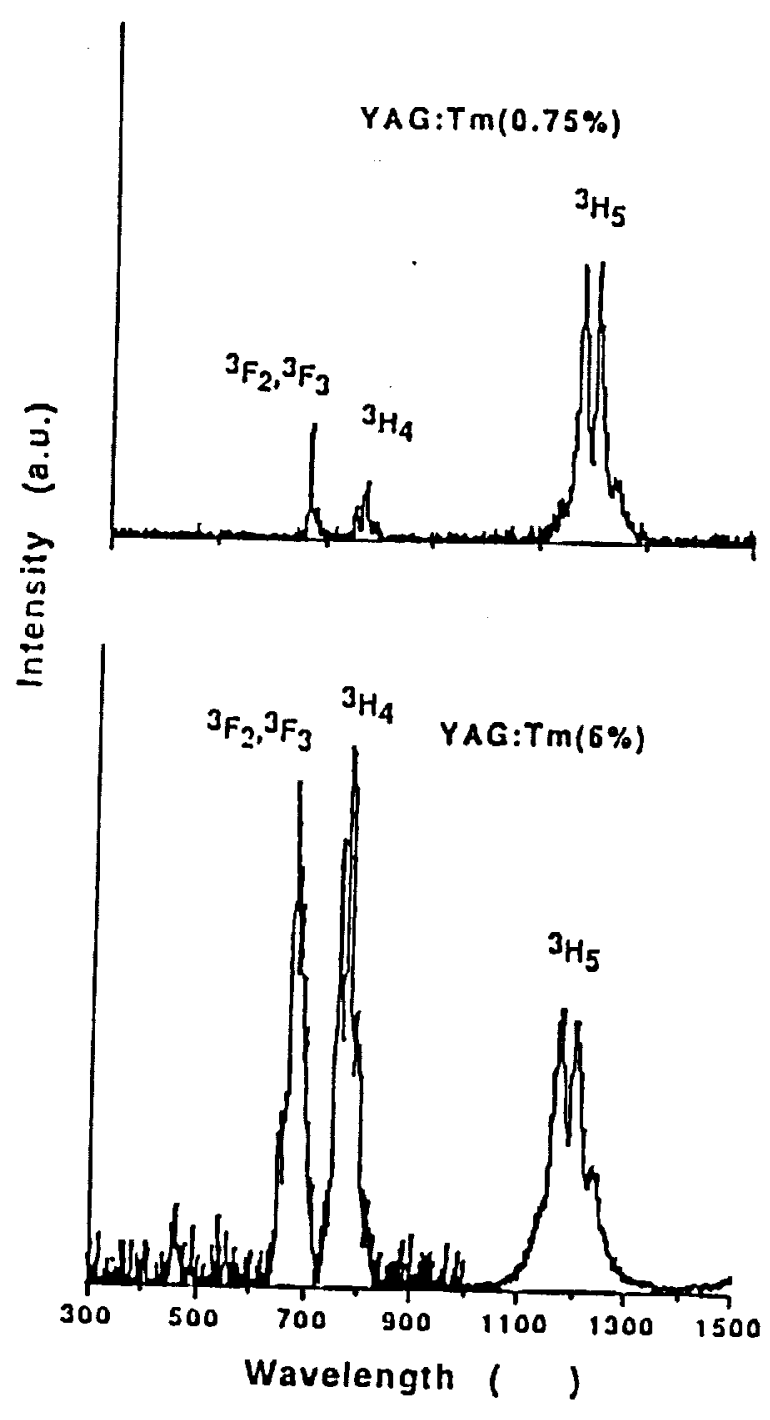

Fig. 3. Excilation spectra of YAG:Tm (0.75\%) and YAG: Tm (1\%) (obtained by monitoring the $1.8 \mu \mathrm{m}$ emission al room lemperature).

a "dip" at $77 \mathrm{~K}$ which becomes more evident at the higher $\mathrm{Tm}$ concentrations.

The decay curves of $\mathrm{Tm}$ emission from the ${ }^{3} \mathrm{~F}_{+}$level represents a rise due to the filling of this level and a long decay. Lifetime of this decay for $\mathrm{Tm}(6 \%)$ sample shown in tig. 8 decreases as temperature varies from 30 to $295 \mathrm{~K}$. 


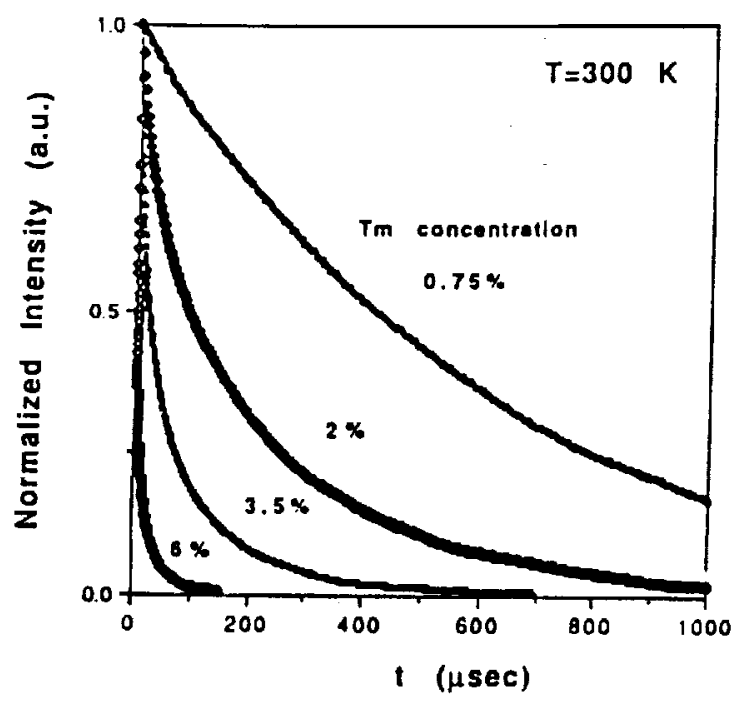

Fig. 4. Decay patierns of the ${ }^{3} \mathrm{H}_{4} \rightarrow{ }^{\prime} \mathrm{H}_{\mathrm{h}}$ emission $(i=830 \mathrm{~nm}$, ior (1) $0.75 \%$. (2) $2 \%$. (3) $3.5 \%$. and (4) $6 \% \mathrm{Tm}$ concentrations at room temperalure The exciting pulses (at $\lambda=785.5 \mathrm{~nm}$ ) were provided by a dye laser.

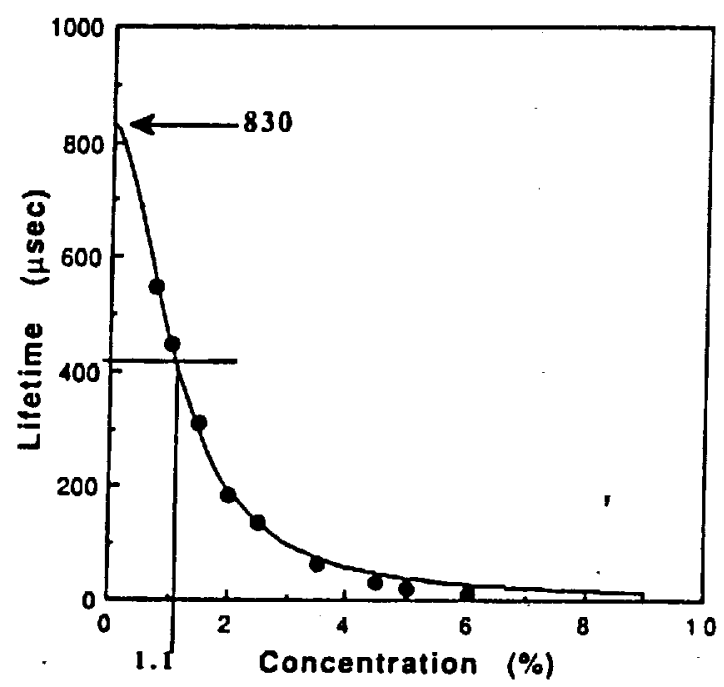

Fig. S. Lifetimes of the ${ }^{3} \mathrm{H}_{4}$ level of $\mathrm{Tm}$ in $\mathrm{Y} . \mathrm{AG}$ as tunction of Tm concentration at room temperature.

\section{Interpretation of results}

\subsection{The process of cross-relaxation}

Cross-relaxation is an interesting case of energy transter among ions in solids. Since the ions involved
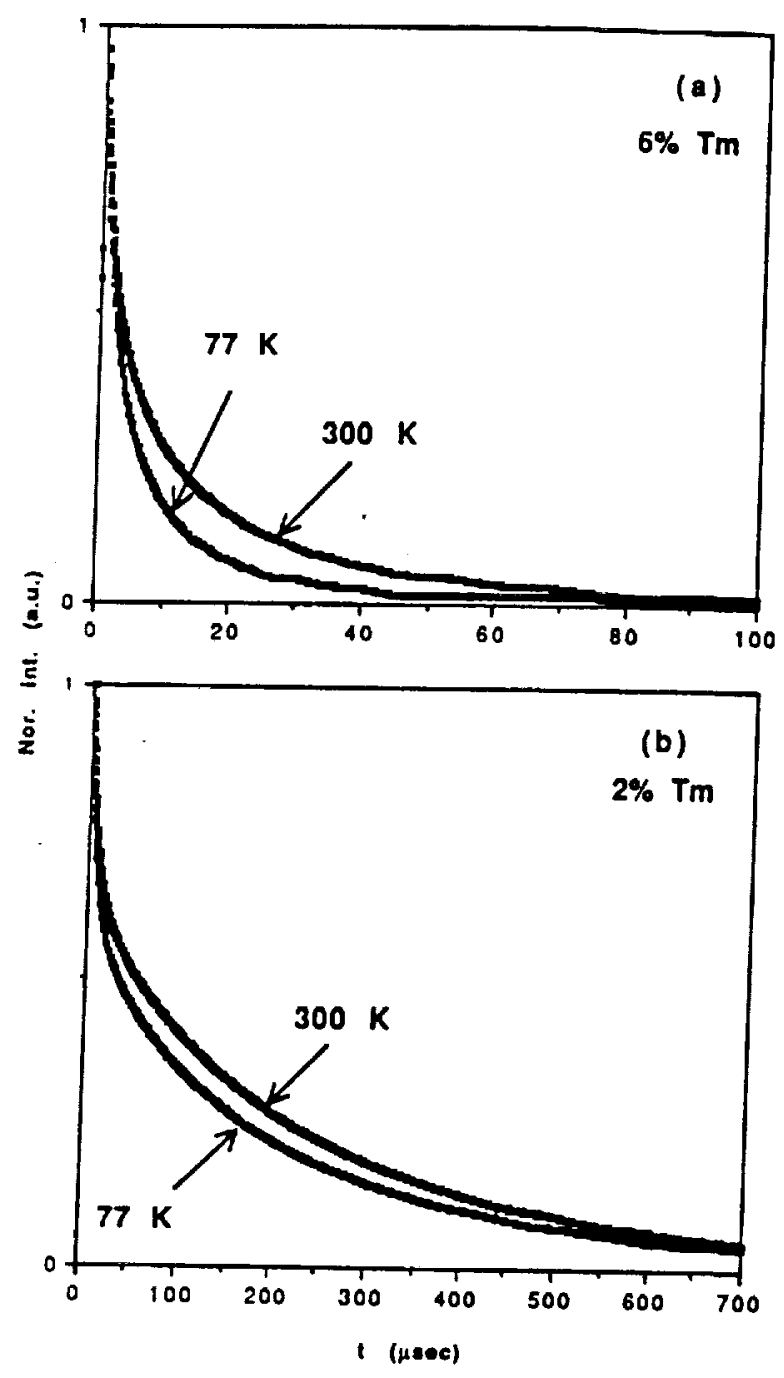

Fig. 6. Decay pattern of the ${ }^{\mathrm{J}} \mathrm{H}_{4}-{ }^{3} \mathrm{H}_{6}$ emission $(i=830 \mathrm{~nm})$ for (a) $0 \%$ and (b) $2 \% \mathrm{Tm}$ concentrations at 300 and $77 \mathrm{~K}$. The exciting pulses (al $i=785.5 \mathrm{~nm}$ ) were provided by a dye laser.

are of the same type. it presents features that are different than those of the case of energy transfer among different ions. The knowledge derived from such studies is relevant to the laser performance of the $\mathrm{Tm}$ ion. by itself or in combination with ions of different types.

The evidence for the existence of a cross-relaxation process ${ }^{3} H_{4} \rightarrow{ }^{3} F_{4} \cdot{ }^{3} H_{6} \rightarrow{ }^{3} F_{4}$ is based on a number of experimental results.

Let us consider first the excitation spectra in fig. 3. The excitation spectrum obtained by monitoring the 


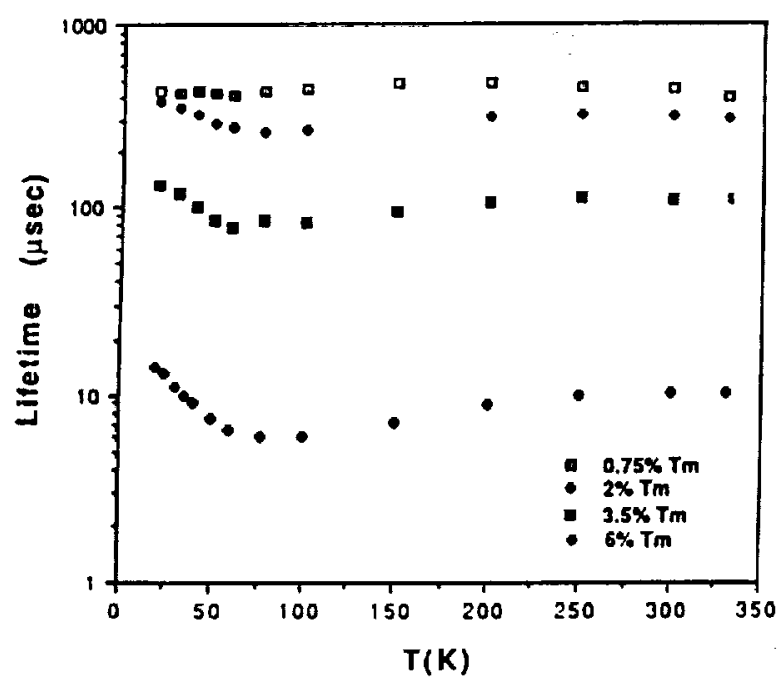

Fig. 7. Lifetimes of the ${ }^{3} \mathrm{H}_{+}$level of $\mathrm{Tm}$ in $\mathrm{YAG}$ as function of temperalure for several $\mathrm{Tm}$ concentrations.

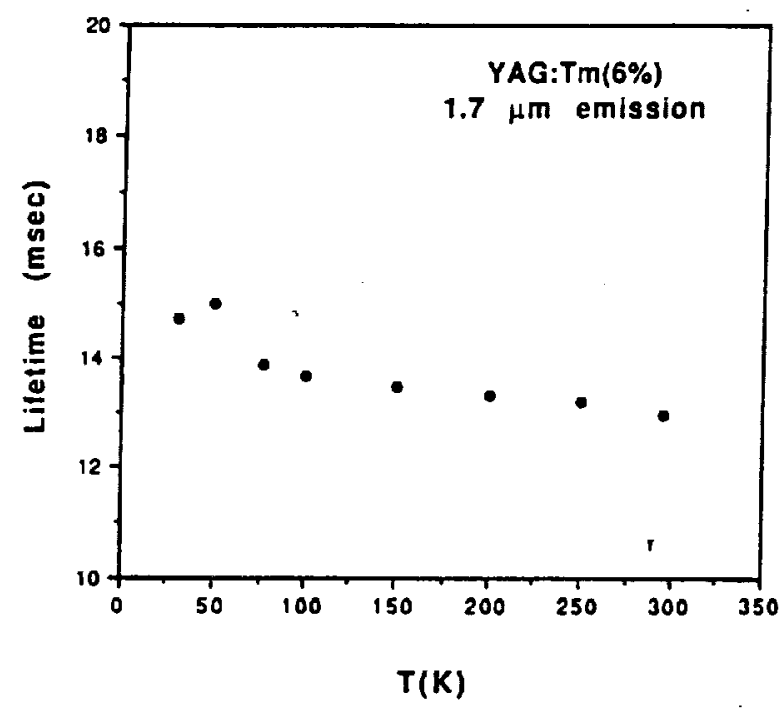

Fig. 8. Lifetimes of the ${ }^{3} F_{4}$ level of $\mathrm{Tm}(6 \%)$ in $Y$ i G as function of temperalure.

$1.8 \mu \mathrm{m}$ emission of $\mathrm{Tm}$ (due to the ${ }^{3} \mathrm{~F}_{4} \rightarrow{ }^{3} \mathrm{H}_{6}$ transition) shows that the emission from the ${ }^{3} F_{4}$ level is present when the system is pumped in the ${ }^{3} F_{2} .{ }^{\prime} F_{1}$. ${ }^{3} H_{4}$, and ${ }^{3} H_{5}$ levels. The data show also that, as the concentration of $\mathrm{Tm}$ increases. the level ${ }^{3} \mathrm{H}_{4}$, and the levels ${ }^{3} F_{2}$ and ${ }^{3} F_{3}$, which are connected to it by fast nonradiative processes, are more and more effective than the level ${ }^{3} \mathrm{H}_{3}$ in pumping the ${ }^{3} \mathrm{~F}_{+}$level. as one would expect in the presence of the cross-relaxation process.

The tifetime data provide additional evidence. The lifetime of the luminescence originating in the ${ }^{3} \mathrm{H}_{4}$ level is quenched as the concentration of $\mathrm{Tm}$ increase. confirming that the cross-relaxation path is available to the $\mathrm{Tm}$ ion in the ${ }^{3} \mathrm{H}_{4}$ level. If the interaction between the $\mathrm{Tm}$ ions responsible for the cross relaxation is of dipole-dipole type. then the lifetime will depend quadratically on the concentration as follows [19]

$\tau=\tau_{0} /\left[1+\left(C / C_{11}\right)^{*}\right]$.

In this expression. $\tau_{0}$ is the intrinsic lifetime of $\mathrm{Tm}$. that is. the lifetime of an isolated $\mathrm{Tm}$ ion. in the absence of any cross-relaxation: $\tau$ is the lifetime of a Tm ion in a sample with $\mathrm{Tm}$ concentration $C$ and it is due both to intrinsic relaxation and cross-relaxation processes: $C_{0}$ is the value of the $\mathrm{Tm}$ concentration at which the lifetime is reduced by half. Fig. 5 shows the dependence of $\mathrm{Tm}$ lifetime on concentration. with the solid curve being the best fit of the data to eq. (1). The intrinsic lifetime of this level determined by extrapolation of the fitted curve to zero concentration is $830 \mu \mathrm{s}$; we used this value to calculate the Tm- Tm cross-relaxation rate. $p$, with the following equation:

$p=\left(\tau^{-1}-10^{-1}\right)^{-1}$.

The cross-relaxation rate from the ${ }^{3} \mathrm{H}_{4}$ level of $\mathrm{Tm}$ at room temperature as function of the square of $\mathrm{Tm}$ concentration is shown in fig. 9.

We derived the temperature dependence of the cross-relaxation by using the formula (2) and the data on the lifetime of the ${ }^{3} \mathrm{H}_{4}$ level at different temperatures. assuming that the intrinsic lifetime $\tau_{0}$ is constant at all temperatures. The results are shown in fig. 10. The rate of the cross-relaxation process is found to increase with increasing $\mathrm{Tm}$ concentration at a given temperature: on the other hand. for a certain $T \mathrm{~m}$ concentration it presents a maximum at $\approx 77 \mathrm{~K}$.

This temperature dependence is due to the changes of the overlap integral between the ${ }^{3} \mathrm{H}_{4} \rightarrow{ }^{3} \mathrm{~F}_{4}$ emission and the ${ }^{3} \mathrm{H}_{n} \rightarrow{ }^{3} \mathrm{~F}_{+}$absorption: this changes are due to the rearrangements of populations in the ${ }^{3} \mathrm{H}_{4}$ and ${ }^{3} \mathrm{H}_{6}$ manifolds. Of course no general conclusion regarding the cross relaxation of $\mathrm{Tm}$ ions should be drawn from this tinding. which depends on the details of the energy level scheme: as matter of fact. in 


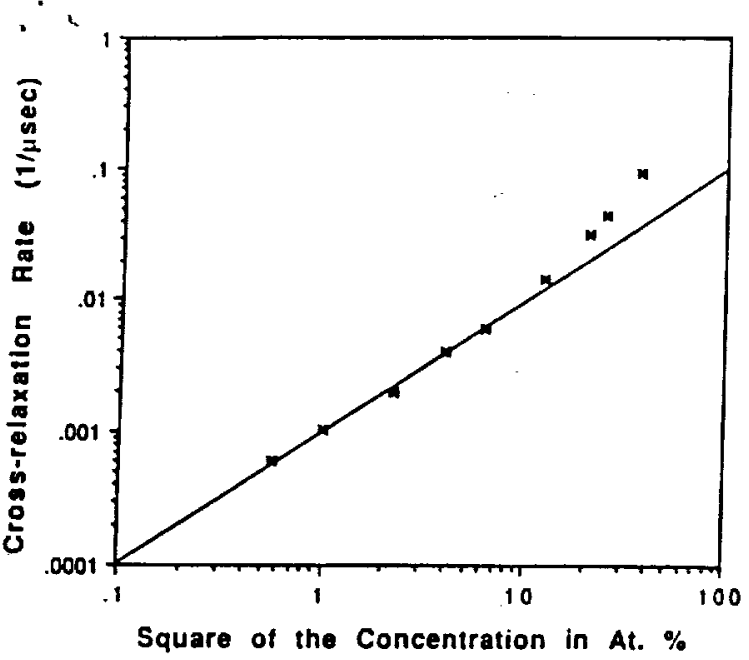

Fig. 9. Cross-relaxation rate among $\mathrm{Tm}$ ions in $\mathrm{YtG}$ as a iunction of the square of the Tm concentration at room temperature.

a similar study of ours of cross relaxation of $\mathrm{Tm}$ ions in the host lattice LiYF 4 . we have found a cross-relaxation rate decreasing with temperature with no maximum in the curve of rate versus temperature [20].

\subsection{Microparameter for cross relaxation}

The rate of the energy transfer between a sensitizer ion (S) and an activator ion (A), $w_{\mathrm{SA}}$, depends on the separation between the ions. $R$. This dependence can be expressed in a multipolar expansion as follows:

${ }^{\prime \prime s+1}(R)=C^{(n)} / R^{n}+C^{(x)} / R^{x}+C^{(1) 1} / R^{\prime \prime)}+\ldots$.

where the first three terms corresponds to dipole-dipole. dipole-quadrupole. and quadrupole-quadrupole interactions. respectively. If there is a dominant multipolar interaction, then the transfer rate assumes the simpler form

$\|{ }_{s+1}(R)=C^{(n)} / R^{n}$.

and we can define an "energy transfer" radius, $R_{0}$, as bollows:

${ }^{\prime \prime S}(R)=C^{(n)} / R^{n}=\tau_{11}^{-1}\left(R_{0} / R\right)^{n}$.

where $\tau_{1}$ is the effective decay time of $S$ in absence of $A$ and $R_{0}$ is the separation between ions at which the energy transfer rate is equal to the decay rate of sensitizer.

The characteristic energy transfer radius $R_{0}$ in the case of dipole-dipole interaction $R_{0}$ is given by [1]

$R_{0}^{b}=\epsilon\left(3 e^{2} / m c^{2} 2^{6} \pi^{4}\right) f_{A} \int \lambda^{6} g_{S}(\lambda) g_{A}(\lambda) \mathrm{d} \lambda$.

In this expression. $\epsilon$ is the quantum efficiency of the donor luminescence (in the absence of any acceptor). that is

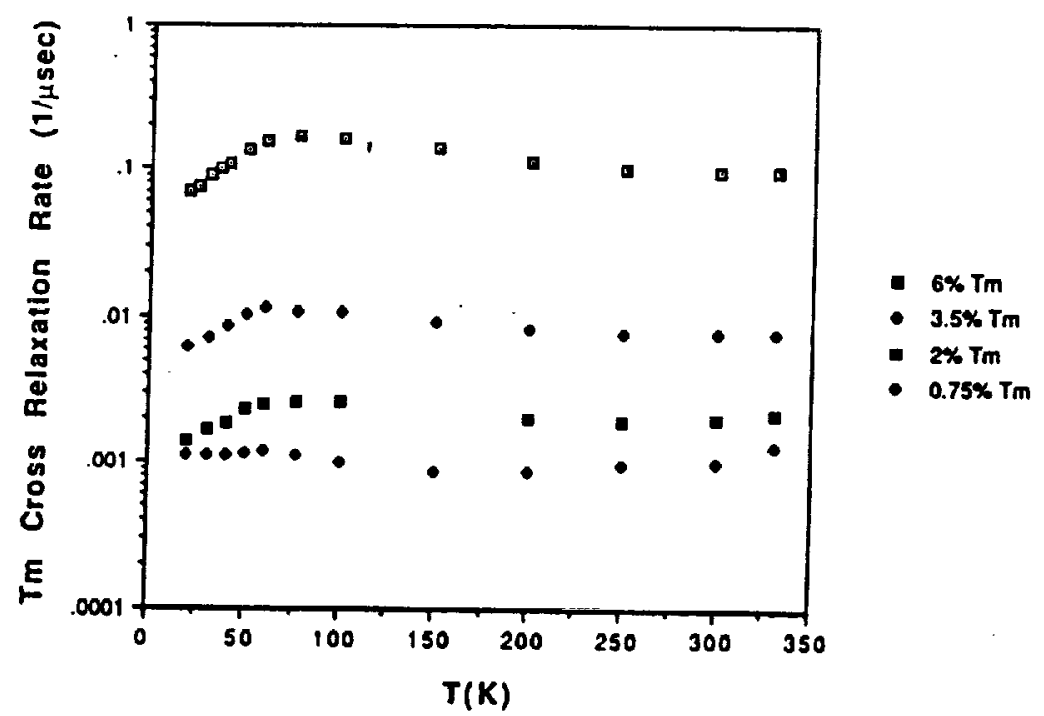

Fig. 10. Cross-relaxation rate among $T m$ ions in $Y A G$ as a tunction of temperature for several $\mathrm{Tm}$ concentrations. 
$\because \quad \cdot$

$\epsilon=($ probability of radiative decay $)$

$X$ ( probability of radiative decay

+ probability of nonradiative decay $)^{-1}$.

The overlap integral involves $g_{\mathrm{s}}(i)$ and $g_{+}(i)$. the normalized emission and absorption data as

$f_{\lambda}=\left(m c^{2} n / \pi e^{i} c_{+}\right) \int\left(1 / \lambda^{2}\right) \alpha_{1}(\lambda) d \lambda$.

Here $n$ is the index of refraction. $c_{+}$and $\alpha_{+}(i)$ are the concentration and the absorption coefficient of the activator, respectively.

The microscopic interaction parameter of eq. (f). given by

$\left.C^{(6)}=R_{n}^{n} / \tau_{0}\right)$

is the probability per unit time of energy transfer when $S$ and $A$ are at the distance of $1 \mathrm{~cm}$. We have calculated this parameter for the ${ }^{3} \mathrm{H}_{4} \rightarrow{ }^{3} \mathrm{~F}_{4},{ }^{3} \mathrm{H}_{6} \rightarrow{ }^{3} \mathrm{~F}_{4}$ crossrelaxation process using the approximation that the concentration of activator ions. $c_{x}$. is the total concentration of $\mathrm{Tm}$ ions.

The spectral data relevant to this calculation are the normalized $\mathrm{Tm}$ emission corresponding to the ${ }^{3} \mathrm{H}_{4} \rightarrow{ }^{3} \mathrm{H}_{4}$ transition and the normalized Tm absorption corresponding to the ${ }^{3} \mathrm{H}_{6} \rightarrow{ }^{3} \mathrm{~F}_{4}$ transition. These data at room temperature are shown in fig. 11 . The various quantities entering the expression for and the value of the microparameter are reported in table 1 . The quantity $\epsilon$ was calculated taking the ratio of the lifetime at room temperature to the lifetime at $30 \mathrm{~K}$.

The time evolution of the emission from a sensitizer's level following a pulsed excitation may provide an additional and independent way of finding a value for the energy transfer microparameter. This is certainly so when no fast diffusion among sensitizers is present. In such a case the decay of the sensitizer is expressed by the following expression [21]:

$$
\begin{aligned}
\rho(t) & =\exp \left[-1 / \tau_{0}\right. \\
& \left.-c_{+} /\left(4 \pi R_{1}^{3} / 3\right) \Gamma(1-3 / n)\left(1 / \tau_{1}\right) 3 / n\right] .
\end{aligned}
$$

where $n$ is either 6 (dipole-dipole). 8 (dipole-quadrupole) or 10 (quadrupole-quadrupole). We can definitely apply this formula to the present case of cross relaxation because. as we shall discuss in the following section 4.3 . the migration of excitation en- argy among the $\mathrm{Tm}$ ions in level ${ }^{\prime} \mathrm{H}_{4}$ is slow compared to cross relaxation [17].

Indeed we have been able to reproduce the curves which appear in fig. 4 by using eq. (10) in which we have substituted the intrinsic lifetime of the ${ }^{3} \mathrm{H}_{4}$ level ( $830 \mu \mathrm{s}$ ) and values of $R_{0}$ close to the one given by the previous calculation and appearing in table 1 . We used the value given in this table. $11 \dot{A}$, to fit the decay curve corresponding to the $3.5 \%$ concentration, and we used the values $8,10.13$ for the curves corresponding to $0.75,2$, and $6 \%$. respectively. The curves that we have generated are reported in fig. 12 and are very close to the experimental curves of fig. 4.

\subsection{Energ! migration among Tin ions in the level ${ }^{3} H_{4}$}

When the $\mathrm{Tm}$ ions are excited to the level ${ }^{3} \mathrm{H}_{4}$, in addition to the cross-relaxation process. a process of migration of the excitation energy among $T m$ ions can in principle take place. How fast is this process in comparison to the cross relaxation? We expect this latter process to be faster for the following reason.

If the migration were very fast the excited Tm ions, being strongly coupled to each other, would form a system with a single decay pattern. The decay curves ( see tig. 4), instead, obey eq. (10). This formula applies to systems with various sensitizers in the crystal having various rates of decay which combine in giving the expression (10). We note also that the rate of this migration has been measured by other workers and found to be much slower than the cross-relaxation rate [17].

\subsection{Energ!' migration among Tin ions in the level ${ }^{3} F_{\text {. }}$}

Migration can also take place when the $\mathrm{Tm}$ ions are residing in level ${ }^{\mathrm{S}} \mathrm{F}_{+}$, following the process of cross relaxation. The decay curves of this emission indicates two main features: a rise due to the cross relaxation and an exponential decay. The lifetime of this decay for $\mathrm{Tm}(6 \%)$ sample as function of temperature is reponed in tig. 8 . We have calculated the microscopic interaction parameter for the related energy Iranster process ${ }^{3} \mathrm{~F}_{4} \rightarrow{ }^{3} \mathrm{H}_{6} \cdot{ }^{3} \mathrm{H}_{6} \rightarrow{ }^{3} \mathrm{~F}_{4}$ by using Dexter theory [1]. 


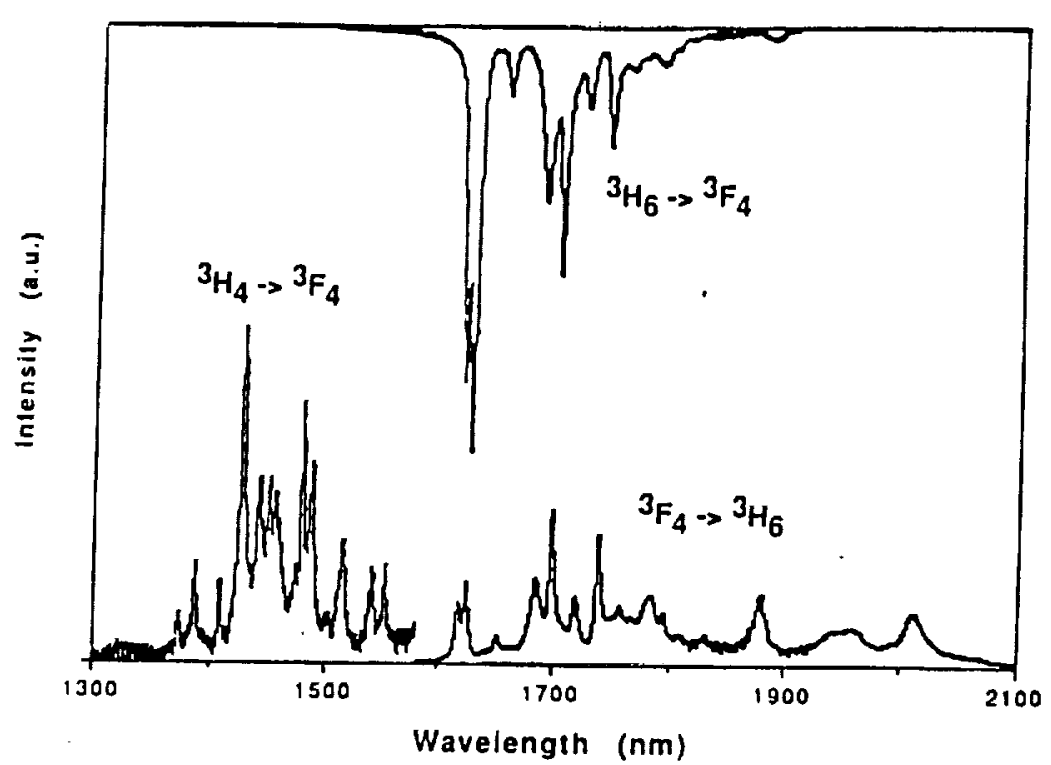

Fig. II. Spectral data used to calculate the overlap integrals relevant to the cross reiaxation and migration processes at room temperature.

Table I

Microparameters of $T m: Y i G$ at room temperalure for (a) cross relaxation ( ${ }^{3} H_{4} \rightarrow{ }^{1} F_{4}:{ }^{3} H_{6} \rightarrow{ }^{1} F_{4}$ ) and (b) migration (among ${ }^{3} F_{4}$ ) processes.

\begin{tabular}{|c|c|c|c|c|c|}
\hline & 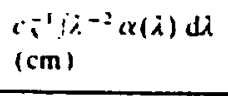 & $\begin{array}{l}\int \lambda^{6} g_{s}(\lambda) g_{+}(\lambda) d u \\
\left(\mathrm{~cm}^{3}\right)\end{array}$ & t & $\begin{array}{l}R_{0} \\
(A)\end{array}$ & $\begin{array}{l}C^{(6)} \\
\left(\mathrm{cm}^{0} / \mathrm{s}\right)\end{array}$ \\
\hline $\begin{array}{l}\text { (a) } \\
\text { (b) }\end{array}$ & $\begin{array}{l}1.29 \times 10^{-11} \\
1.29 \times 10^{-18}\end{array}$ & $\begin{array}{l}0.62 \times 10^{-30} \\
75 \times 10^{-20}\end{array}$ & $\begin{array}{c}0.83 / 0.83 \\
13 / 15\end{array}$ & $\begin{array}{l}11.4 \\
25\end{array}$ & $\begin{array}{l}0.27 \times 10^{-36} \\
1.7 \times 10^{-36}\end{array}$ \\
\hline
\end{tabular}

$\left(3 n / 2^{6} \pi^{5}=2.7 \times 10^{-4}\right)$

The spectral data relevant to this calculation are the normalized $\mathrm{Tm}$ emission corresponding to the ${ }^{3} \mathrm{~F}_{4} \rightarrow{ }^{3} \mathrm{H}_{6}$ transition and the normalized $T \mathrm{~m}$ absorption corresponding to the ${ }^{3} \mathrm{H}_{6} \rightarrow{ }^{3} \mathrm{~F}_{+}$transition. These data at room temperature are shown in fig. 11 . The various quantities entering the expression for and the value of the microparameter are reported in table !.

French and Powell have used laser-induced grating spectroscopy to measure spátial energy migration without spectral transter [22] by using the four-wave mixing technique. They report data from which we can derive a value for $R_{t}$ of $\sim 100 \dot{A}$ : this value is expected to be larger than the one derived by using the Dexter theory [1.23] and dealing with partial overlap.

\section{Conclusions}

On the basis of our experimental observations and of our related calculations we can present the following conclusions:

(i) We have found additional evidence that supports the model proposed by other workers for the dynamics of $\mathrm{Tm}$ ions following their excitation to the ${ }^{3} \mathrm{H}_{4}$ level (see, for example, ref. [4]). According to this model, excitation of an ion to this level is followed by a fast cross-relaxation process whose rate increases with concentration and is temperature dependent with a maximum value at $77 \mathrm{~K}$.

(ii) Another inter-ionic process may transfer the excitation of a particular ion in the ${ }^{3} \mathrm{H}_{4}$ level to a different $\mathrm{Tm}$ ion. thus producing a migration of the excitation energy. This process is much slower than the cross-relaxation process. 


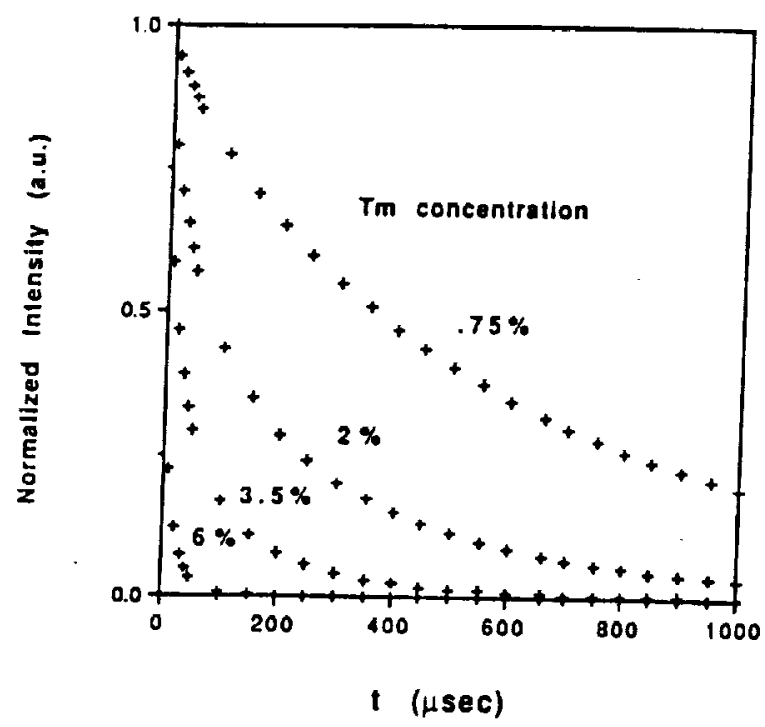

Fig. 12. Curves of sensitizer $\left({ }^{3} \mathrm{H}_{4}\right)$ decay obtained by using formula (10).

(iii) Following the cross relaxation of a $\mathrm{Tm}$ ion to the ${ }^{3} \mathrm{~F}_{\downarrow}$ level a migration of this excitation sets in. The rate of such process is found to be greater than that of cross relaxation and. because of it, the excitation energy diffuses easily among the $\mathrm{Tm}$ ions. a very useful condition if. as it may be the case. $\mathrm{Tm}$ is used to sensitize other ions. such as Ho. We note here that the result obtained by other workers [23] and by us [24] indicate that. for the usual condition used in laser applications of much larger $\mathrm{Tm}$ than Ho concentration. the migration of the ${ }^{3} F_{4}$ excitation energy is faster than the energy transter between $\mathrm{Tm}$ and $\mathrm{Ho}$ ions.

(iv) We have measured the microparameter associated with the cross relaxation process and the microparameter associated with the migration of ${ }^{3} \mathrm{~F}_{4}$ excitation energy. In particular the former has been obtained by using two different kind of data: the spectral data and the details of the kinetics of the cross relaxing level. The latter has been obtained by using solely spectral data. We trust that, besides providing additional evidence for the existing model. we have put it on a more quantitative basis.

\section{Acknowledgements}

We would like to thank C.H. Bair. P. Brockman and R.V. Hess for valuable discussions. C.W. Edwards.
A.T. Inge and E.A. Modlin for their help with the experiments. This work was supported by NASA Grants NAG-1-796 and NAG-1-955.

\section{References}

[1] D.L. Dexter. J. Chem. Phys. 212 (1953) 836, Phys. Rev. 108 (1957) 630.

[2] A..t. Kaminskii. Laser crystals. their physics and propenies (Springer. Berlin. 1981. 1990).

[3] L.F. Johnson. J.E. Geusic and L.G. Van Uitert. Appl. Phys. LitI. \& (1966) 200.

[H] T.Y. Fan. G. Huber, R.L. Byer and P Mitzscherlich, IEEE J. Quantum Electronics 24 (1988) 924.

[5] G. Ammagan. B. Di Barolo and A.M. Buoncristiani. J. Luminescence 44 (1989) 129.

[6] G. Armagan. B. Di Banolo and A.M. Buoncrisliani. J. Luminescence 44 (1989) 141.

[7] L.F. Johnson. J.E. Geusic and L.G. van Uitert. Appl. Phys. Lett. 7 (1965) 127.

[8] B.M. Antipenko, A.S. Glebov, T.I. Kiseleva and V.A. Pismennyi. Opt. Spectr. (USSR) 63 (1987) 230.

[9] J.K. Tyminsky, D.M. Franich and M. Kokta. J. Appl. Phys. 65 (1989) 15 .

[10] G.J. Kiniz. L. Esterowitz and R. Allen. Electron. Lelt. 23 (1987) 616.

[11] A. Brenier. J. Rubin. R. Moncorgè and C. Pedrini, J. Phys. (France) 50 (1989) 1463.

[12] B.M. Antupenko. A.S. Glebov, T.I. Kiseleva and V.A. Pismennyi. Opt. Spectr. (USSR) 60 (1986) 95.

[13] G.J. Kiniz. R. Allen, and L. Esterowitz. Posideadline Papers. Conf. Laser Electro-Opt. (Optical Society America. Washingion DC 1987).

[ 14 ] G. Armagan. A.M. Buoncristiani, W.C. Edwards, A.T. Inge and B. Di Bartolo. OSA Proc. on Advanced solid state lasers. Vol. 6. eds. H.P. Jenssen and G. Dubé (Optical Sociely of America. Washington DC 20036, 1990) p. 144.

[15] G. Amagan. A.M. Buoncristiani and B. Di Banolo. J. Luminescence $48 \& 49$ (1991) 171 .

[16] J.B. Gruber. M.E. Hills, R. Macfarlane, C.E. Morrison, G.A. Tumer. G.J. Quarles. G.J. Kintz and L. Esterowitz, Phys. Rev. B 40 ( 1989 ) 9464.

[17] T. Becker. R. Clausen. G. Huber. E. Duczynski and P. Mitzscherlich. OSA Proc. On Advanced solid state lasers. Vol. $S$ eds. M.L. Shand and H.P. Jenssen (Optical Society of America. Washington. DC 20036. 1989) p. 154.

(18] D.L. Dexter and J.H. Schulman. J. Chem. Phys. 22 (1954) 1063.

[19] G. Armagan. M. Buoncristiani, A.T. Inge and B. Di Bartolo. Advanced Solid State Laser Conf.. Hilton Head.. S.C.. March. 1991, paper TuC3-1.

[20] M.Inokuti and F. Hirayama. J. Chem. Phys. 43 (1963) 1978.

[21] V.A. French and R.C. Powell. Optics Let1. 16 (1991) 666.

[22] R.C. Powell. private communication.

[2J]G. Amagan. M. Buoncristiani and B. Di Bartolo, to be published. 